\title{
Overtreatment of Nonneoplastic Gallbladder Polyps due to Inadequate Routine Ultrasound Assessment
}

\author{
Sarah Z. Wennmacker ${ }^{a}$ Elise AJ. de Savornin Lohman ${ }^{a}$ Nesar A. Hasami ${ }^{a}$ \\ Iris D. Nagtegaal ${ }^{b}$ Marja A. Boermeester ${ }^{c} \quad$ Joanne Verheij $^{d}$ \\ Ernst Jan Spillenaar Bilgen ${ }^{\mathrm{e}}$ Jos W.H. Meijer ${ }^{f}$ Koop Bosschag $^{g}$ \\ Johannes C. van der Linden ${ }^{\mathrm{h}}$ John J. Hermans ${ }^{i}$ Philip R. de Reuver ${ }^{\mathrm{a}}$ \\ Joost P.H. Drenth ${ }^{j}$ Cornelius J.H.M. van Laarhoven ${ }^{a}$
}

\begin{abstract}
${ }^{a}$ Department of Surgery, Radboud University Medical Center, Nijmegen, The Netherlands; ${ }^{b}$ Department of Pathology, Radboud University Medical Center, Nijmegen, The Netherlands; 'Department of Surgery, Academic Medical Center, Amsterdam, The Netherlands; 'Department of Pathology, Academic Medical Center, Amsterdam, The Netherlands; 'Department of Surgery, Rijnstate Hospital, Arnhem, The Netherlands; fDepartment of Pathology, Rijnstate Hospital, Arnhem, The Netherlands; ${ }^{9}$ Department of Surgery, Jeroen Bosch Hospital, 's-Hertogenbosch, The Netherlands; ' Department of Pathology, Jeroen Bosch Hospital, 's-Hertogenbosch, The Netherlands; 'Department of Radiology and Nuclear Medicine, Radboud University Medical Center, Nijmegen, The Netherlands; jDepartment of Gastroenterology and Hepatology, Radboud University Medical Center, Nijmegen, The Netherlands
\end{abstract}

\section{Keywords}

Gallbladder polyp - Ultrasound - Sensitivity and specificity · Neoplasia

\begin{abstract}
Background: The primary aim was to assess the diagnostic accuracy of routine ultrasound assessment for gallbladder polyps. The secondary aim was to identify the characteristics that differentiate neoplastic polyps from nonneoplastic polyps. Methods: A total of 156 patients with histopathologically proven gallbladder polyps in 4 Dutch hospitals between 2003 and 2013 were included. Sensitivity and specificity of ultrasound for polyp size, number of polyps, and polyp type were assessed using histopathological findings as a reference standard. In addition, diagnostic accuracy of sonographic size $\geq 1 \mathrm{~cm}$ for neoplasia was assessed. Subgroup
\end{abstract}

analysis for patients with polyps as primary indication for cholecystectomy was performed. The sonographic polyp characteristics on preoperative routine ultrasound were described. Results: Fifty-six percent of gallbladder polyps were preoperatively identified on ultrasound, of which 31\% were neoplastic. Sensitivity and specificity of ultrasound to estimate polyp size were 93 and 43\% (subgroup; 92 and 33\%). Sensitivity and specificity of sonographic polyp size $\geq 1 \mathrm{~cm}$ for neoplasia were 86 and 32\% (subgroup; 94 and 26\%). No specific sonographic characteristics for neoplastic polyps could be established due to lack of reporting. Conclusion: Routine ultrasound assessment of polyps is associated with overestimation of polyp size and low specificity of sonographic size $\geq 1 \mathrm{~cm}$ for neoplasia, which contributes to surgical overtreatment of nonneoplastic polyps.

(c) 2020 The Author(s)

Published by S. Karger AG, Basel karger@karger.com www.karger.com/dsu

Karger $\stackrel{\text { ' }}{5}$

BOPEN ACCESS
(C) 2020 The Author(s)

Published by S. Karger AG, Basel

This is an Open Access article licensed under the Creative Commons Attribution-NonCommercial-4.0 International License (CC BY-NC) (http://www.karger.com/Services/OpenAccessLicense), applicable to the online version of the article only. Usage and distribution for commercial purposes requires written permission.
Sarah Z Wennmacker

Department of Surgery, Radboud University Medical Center PO Box 9101

NL-6500 Nijmegen (The Netherlands) sarah.wennmacker@ radboudumc.nl 


\section{Introduction}

Neoplastic gallbladder polyps should be managed by cholecystectomy to halt or prevent the development of gallbladder cancer, which is a highly lethal tumor [1]. However, the preoperative differentiation of neoplastic polyps from nonneoplastic polyps remains a diagnostic challenge.

Recently revised international guidelines recommend ultrasonography to identify polyps and evaluate polyp size. Cholecystectomy is advocated for all polyps $\geq 1 \mathrm{~cm}$ in view of the elevated risk of neoplasia of these polyps [2-4]. The validity of this surgical threshold can be questioned. Between one- and two-thirds of polyps with a diameter $\geq 1 \mathrm{~cm}$ are nonneoplastic on postoperative histopathological examination, while one-third of neoplasms are detected in smaller polyps $(<1 \mathrm{~cm})$ [5-7]. A polyp size of $1 \mathrm{~cm}$ could be an inappropriate threshold for neoplasia [7], or preoperative ultrasound may be inaccurate in establishing polyp size $[6,8]$. Both can result in poor patient selection for cholecystectomy in patients with gallbladder polyps. Improved preoperative differentiation between neoplastic and nonneoplastic polyps is crucial for preventing both unnecessary cholecystectomy and the undertreatment of neoplastic gallbladder polyps.

Several studies have suggested that more detailed sonographic characteristics could aid in the preoperative differentiation of polyp types [6, 9-12]. However, the most recent European guidelines do not advise on the characteristics that should be evaluated. Only "comet tail" artifacts, which are seen in some nonneoplastic adenomyomatosis and cholesterol polyps, are given as a criterion for nonneoplastic polyps [4].

The primary aim of the present study was to assess the diagnostic accuracy of routine ultrasound assessment for gallbladder polyp size, number, and neoplastic differentiation, and determine the diagnostic accuracy of the sonographic $1 \mathrm{~cm}$ threshold for neoplasia. Furthermore, this study sought to evaluate the sonographic characteristics that differentiate neoplastic and nonneoplastic polyps.

\section{Methods}

\section{Study Design and Patient Selection}

A retrospective analysis of routine ultrasound reports of all patients with histopathologically proven gallbladder polyps in 2 academic and 2 affiliated teaching hospitals in the Netherlands was conducted. Eligible patients were identified through the Dutch nationwide network and registry of histopathology and cytopathol- ogy (PALGA). The search term "gallbladder" combined with "polyp" or "adenoma" or "adenomatous polyp" or "hyperplastic polyp" or "dysplasia" or "cholesterolosis" or "all benign neoplasms" or "all primary malignancies (incl. cis)" or the individual search term "cholesterol polyp" were used [13]. At the time of the search, PALGA covered the full pathology reports of all pathology laboratories in the Netherlands between 1991 and 2013. All patients with a gallbladder polyp or (focal) wall thickening of the gallbladder $>5 \mathrm{~mm}$ on histopathological evaluation after cholecystectomy between 2003 and 2013 were included, irrespective of the indication for cholecystectomy (because this is not listed in PALGA). Patients who underwent cholecystectomy during more extensive abdominal surgery (e.g., hepatectomy or pancreatectomy) and biopsy results were excluded. Preoperative ultrasound reports and indication for surgery were collected from patients' medical records. $\mathrm{Pa}$ tients who did not undergo preoperative ultrasound were excluded. Details of polyp characteristics were extracted from pathology reports and ultrasound reports.

Based on the pathology reports provided by PALGA, all patients were categorized as diagnosed with either neoplastic polyps ([cyst]adenoma, adenocarcinoma, or other malignancy) or nonneoplastic polyps (all other types of polyps). If a patient had both neoplastic and nonneoplastic gallbladder polyps, they were classified as neoplastic.

\section{Outcomes}

The primary outcomes were the sensitivity and specificity of ultrasound for polyp size $(<1 \mathrm{~cm}$ vs. $\geq 1 \mathrm{~cm})$, number of polyps (single vs. multiple), and polyp type (neoplastic vs. nonneoplastic). The histopathological polyp size, number of polyps, and polyp type were used as reference standard. In addition, the diagnostic accuracy of sonographic polyp size $\geq 1 \mathrm{~cm}$ for neoplasia was assessed. The outcomes were calculated for the full cohort and a subgroup of patients with gallbladder polyps as a primary indication for cholecystectomy.

The secondary outcomes were the sonographic characteristics of neoplastic and nonneoplastic polyps described on preoperative ultrasound. These characteristics included size, number, echogenicity, internal pattern, shape and surface of the polyps, and invasion of the liver.

\section{Statistical Analysis}

The sensitivities and specificities were calculated using $2 \times 2$ tables and were displayed in forest plots. A comparison of sonographic and histopathological polyp size was illustrated using McNemar's test. The sonographic characteristics were reported for patients with neoplastic and nonneoplastic polyps. Dichotomous variables were summarized as the percentage of the total number of patients for whom information on the variable was available. Continuous variables were presented as mean values with SD, and footnotes were used to report the total number of patients for whom information on the variable was available. The associations between characteristics and polyp type (neoplastic vs. nonneoplastic) were assessed using univariate logistic regression analysis. All variables with a $p$ value $<0.1$ were included in a multivariate logistic regression analysis with backward elimination, retaining gender and age as covariates. A $p$ value $<0.05$ was considered statistically significant. All statistical analyses were conducted using SPSS Statistics 22.0 (IBM). 


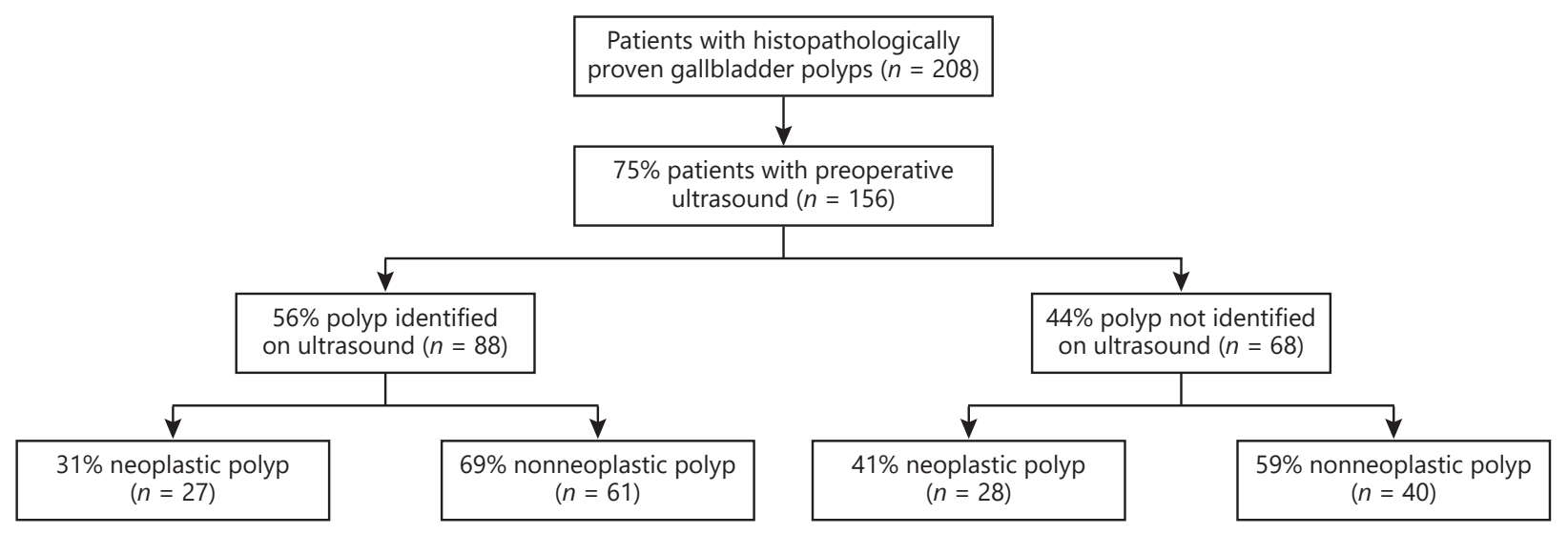

Fig. 1. Flow diagram of patient inclusion and polyp type.

\begin{tabular}{|c|c|c|c|c|c|c|c|c|}
\hline a Full Cohort & TP & FP & FN & $\mathrm{TN}$ & Sensitivity $(95 \% \mathrm{Cl})$ & Specificity $(95 \% \mathrm{Cl})$ & Sensitivity $(95 \% \mathrm{Cl})$ & Specificity $(95 \% \mathrm{Cl})$ \\
\hline Size $(\geq 1 \mathrm{~cm})$ & 26 & 12 & 2 & 9 & $0.93[0.76,0.99]$ & $0.43[0.22,0.66]$ & $\longrightarrow$ & - \\
\hline Single polyp & 48 & 5 & 12 & 18 & $0.80[0.68,0.89]$ & $0.78[0.56,0.93]$ & $\rightarrow-$ & $\longrightarrow-$ \\
\hline \multirow[t]{2}{*}{ Neoplastic polyp } & 12 & 3 & 1 & 6 & $0.92[0.64,1.00]$ & $0.67[0.30,0.93]$ & $\longrightarrow$ & $\longrightarrow$ \\
\hline & & & & & & & $\begin{array}{lllllll} & & & 1 & & \\
0 & 0.2 & 0.4 & 0.6 & 0.8 & 1.0\end{array}$ & $\begin{array}{llllll} & 1 & & 1 & 1 & \\
0 & 0.2 & 0.4 & 0.6 & 0.8 & 1.0\end{array}$ \\
\hline b Subgroup & TP & FP & FN & TN & Sensitivity $(95 \% \mathrm{Cl})$ & Specificity $(95 \% \mathrm{Cl})$ & Sensitivity $(95 \% \mathrm{Cl})$ & Specificity $(95 \% \mathrm{Cl})$ \\
\hline Size $(\geq 1 \mathrm{~cm})$ & 24 & 12 & 2 & 6 & $0.92[0.75,0.99]$ & $0.33[0.13,0.59]$ & $\longrightarrow$ & $\longrightarrow$ \\
\hline Single polyp & 35 & 4 & 11 & 14 & $0.76[0.61,0.87]$ & $0.78[0.52,0.94]$ & $\rightarrow-$ & $\longrightarrow$ \\
\hline \multirow[t]{2}{*}{ Neoplastic polyp } & 11 & 3 & 1 & 4 & $0.92[0.62,1.00]$ & $0.57[0.18,0.90]$ & $\longrightarrow$ & $\rightarrow-$ \\
\hline & & & & & & & $\begin{array}{lllllll}0 & & & 1 & 1 & 1 \\
0 & 0.2 & 0.4 & 0.6 & 0.8 & 1.0\end{array}$ & $\begin{array}{llllll} & 1 & & 1 & 1 & \\
0 & 0.2 & 0.4 & 0.6 & 0.8 & 1.0\end{array}$ \\
\hline
\end{tabular}

Fig. 2. Diagnostic accuracy of ultrasound for polyp size, number, and polyp type. a Full cohort. $\mathbf{b}$ Subgroup of patients with gallblad-

\section{Results}

Two-hundred and eight patients with a histopathologically proven gallbladder polyp through PALGA were identified. A total of 156/208 patients (75\%) who underwent preoperative ultrasound were included in this study. A total of 55/156 patients (35.3\%) had a neoplastic polyp (average histopathological size $24.5 \mathrm{~mm}$ ), and 101/156 patients $(64.7 \%)$ had a nonneoplastic polyp (average histopathological size $8.6 \mathrm{~mm}$ ). The mean age of the cohort was 56.8 years (SD 13.8), and 113/156 patients (72.4\%) were female.

In 68/156 patients (43.6\%), no polyps were detected on preoperative ultrasound. The indication for cholecystec- der polyps as primary indication for cholecystectomy. TP, true positive; FP, false positive; FN, false negative; TN, true negative. tomy in these 68 patients was symptomatic cholecystolithiasis in 64 patients $(94.1 \%$; including a history of complicated cholecystolithiasis in 13\%), 2 had biliary obstruction e.c.i. (2.9\%), 1 had acalculous cholecystitis (1.5\%), and 1 had abdominal symptoms e.c.i. (1.5\%). In 88/156 patients $(56.4 \%), 1$ or more polyps were preoperatively identified on ultrasound. These included $27 / 55$ neoplastic polyps (49.1\%) and 61/101 nonneoplastic polyps (60.4\%; $p=0.17)$. A flowchart of patient inclusion and type of included polyps is shown in Figure 1.

Polyp size was reported in 65/88 patients (73.8\%). The number of polyps in all patients and polyp type was specified in $27 / 88$ patients $(30.7 \%)$ based on a polyp size $\geq 1$ 


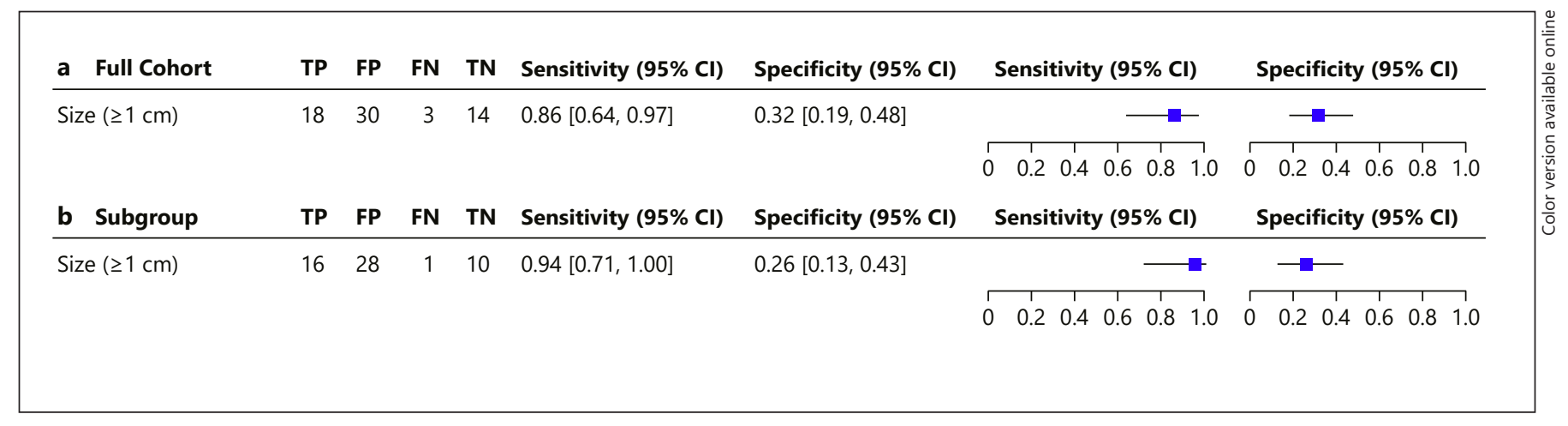

Fig. 3. Diagnostic accuracy of sonographic polyp size $\geq 1 \mathrm{~cm}$ for neoplastic polyp type. a Full cohort. b Subgroup of patients with gallbladder polyps as primary indication for cholecystectomy. TP, true positive; FP, false positive; FN, false negative, TN, true negative.

size was significantly larger in neoplastic polyps on univariable analysis ( 26.9 vs. $13.1 \mathrm{~mm}, p=0.05$ ), though was not significant in the multivariable analysis. Due to a lack of reporting, analysis of the more detailed sonographic characteristics was not conducted.

\section{Discussion}

This study illustrates the shortcomings of routine ultrasound assessment for gallbladder polyps: a low sensitivity for gallbladder polyp identification, low specificity for polyp size estimation, and low specificity of the sonographic 1-cm threshold for neoplasia. Additionally, detailed characteristics predictive of polyp type are not routinely evaluated.

A sensitivity of $56 \%$ for polyp identification is low compared with a recent Cochrane review that showed an overall sensitivity of $84 \%$ [14]. However, the results of individual studies included in this review varied from 45 to $100 \%$, illustrating the large heterogeneity of the studies. Although $1 \mathrm{~cm}$ is the best cutoff size for indicating neoplasia in gallbladder polyps $[1,7,15]$, a systematic review by Babu et al. [16] has shown large heterogeneity in the diagnostic accuracy of ultrasound for establishing polyp size. The summarized sensitivities varied from 78 to $100 \%$ and specificities from 52 to $87 \%$ [16]. The present study showed a comparable sensitivity but a lower specificity of only $26-32 \%$.

Small cohorts and the development of ultrasound equipment in the study periods could explain the heterogeneity of the results. The different attitudes toward the detection and evaluation of polyps during ultrasound as- 
Table 1. Sonographic characteristics of gallbladder polyps

\begin{tabular}{|c|c|c|}
\hline & $\begin{array}{l}\text { Neoplastic } \\
\text { (27 patients) }\end{array}$ & $\begin{array}{l}\text { Nonneoplastic } \\
\text { (61 patients) }\end{array}$ \\
\hline \multicolumn{3}{|l|}{ Polyp size, $\mathrm{mm}$} \\
\hline Mean $^{\#}(\mathrm{SD})$ & $26.9(41.3)$ & $13.1(13.7)^{*}$ \\
\hline$\geq 10 \mathrm{~mm}, n / n(\%)$ & $18 / 21(85.7)$ & $30 / 44(68.2)^{* *}$ \\
\hline Single polyp, $n / n(\%)$ & $20 / 27(74.1)$ & $34 / 61(55.7)^{* * *}$ \\
\hline \multicolumn{3}{|l|}{ Shape } \\
\hline Pedunculated, $n / n$ & $1 / 1$ & $3 / 4$ \\
\hline Sessile, $n / n$ & & $1 / 4$ \\
\hline \multicolumn{3}{|l|}{ Surface } \\
\hline Irregular, $n / n$ & $3 / 3$ & $3 / 5$ \\
\hline Smooth, $n / n$ & & $2 / 5$ \\
\hline \multicolumn{3}{|l|}{ Echogenicity } \\
\hline Hypoechogenic, $n / n$ & $1 / 3$ & $2 / 5$ \\
\hline Hyperechogenic, $n / n$ & $2 / 3$ & $3 / 5$ \\
\hline \multicolumn{3}{|l|}{ Internal pattern } \\
\hline Homogeneous, $n / n$ & & $1 / 8$ \\
\hline Heterogeneous, $n / n$ & $1 / 5$ & \\
\hline Cysts, $n / n$ & $2 / 5$ & $3 / 8$ \\
\hline Hyperechogenic spots, $n / n$ & $2 / 5$ & $4 / 8$ \\
\hline Invasion in liver, $n / n$ & $3 / 3$ & $0 / 3$ \\
\hline
\end{tabular}

\# Sixty-five patients. ${ }^{*} p=0.05$, not significant in multivariate analysis. ${ }^{* *} p=0.13$. ${ }^{* * *} p=0.10$, not significant in multivariate analysis.

sessment in the context of a clinical trial compared with clinical practice could also be a contributing factor [17, 18]. Studies with lower sensitivities generally incorporate data from routine clinical practice, as opposed to data gathered specifically for research purposes. This was recently discussed in other studies that showed a sensitivity of just $8.3 \%$ for polyp identification and a specificity of $53 \%$ for the $1-\mathrm{cm}$ surgical threshold in routine clinical practice $[19,20]$.

The shortcomings in the diagnostic accuracy of routine ultrasound assessment directly impact clinical practice, as indication for cholecystectomy for gallbladder polyps is primarily based on ultrasound results. Due to the present study design, all patients with gallbladder polyps on histopathology after cholecystectomy were identified, including patients without preoperative sonographic identification of polyps who underwent cholecystectomy for a different indication (e.g., symptomatic gallstones). In these symptomatic patients, the false negative ultrasound result did not have implications for clinical practice. The potential undertreatment of neoplastic
Table 2. Overview of imaging characteristics for gallbladder polyps on ultrasound

\begin{tabular}{l} 
- Polyp size $^{\mathrm{a}}$ \\
\hline - Number of polyps ${ }^{\mathrm{b}}$ \\
\hline - Mobility \\
\hline - Acoustic shadowing \\
\hline - Reverberation "comet tails"e \\
\hline - Echogenicity (hypo-, iso-, or hyperechogenic) \\
\hline - Internal pattern (homo- or heterogeneous) \\
\hline - Specific features (hyperechogenic spots, microcysts, \\
microcalcifications) \\
\hline - Shape (pedunculated, sessile) \\
\hline - Surface (smooth, irregular, nodular) \\
\hline - Gallbladder wall invasion \\
\hline - Vascularity
\end{tabular}

a Zielinski, J Gastrointst Surg, 2008; Kim, Eur Radiol, 2016; Wiles, Eur Radiol, 2017; Liu, J Gastroenterol Hepatol, 2018; Kim, EurRadiol,2018; Miwa, Abdom Radiol (NY), 2019; Smereczynski,

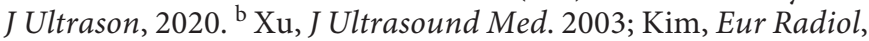
2016; Kim, Eur Radiol, 2018; Smereczynski, J Ultrason, 2020. c Wiles, Eur Radiol, 2017; Smereczynski, J Ultrason, 2020. d Wiles, Eur Radiol, 2017; Smereczynski, J Ultrason, 2020. e Sugiyama, Gut, 2000; Wiles, Eur Radiol, 2017; Golse, J Visc Surg, 2017. f Azuma, Am J Surg, 2001; Cheon, World J Gastroenterol, 2009; Kim, Eur Radiol, 2016; Kim, Eur Radiol, 2018; Miwa, Abdom Radiol (NY), 2019. ' Azuma, Am J Surg, 2001; Cheon, World J Gastroenterol, 2009; Kim, Eur Radiol, 2018; Miwa, Abdom Radiol (NY), 2019. ' h Sugiyama, Gut, 2000; Azuma, Am J Surg, 2001; Cheon, World J Gastroenterol, 2009; Liu, J Gastroenterol Hepatol, 2018; Kim, Eur Radiol, 2018. i Sugiyama, Gut, 2000; Azuma, Am J Surg, 2001; Xu, J Ultrasound Med, 2003; Cheon, World J Gastroenterol, 2009; Kim, Eur Radiol, 2016; Wiles, Eur Radiol, 2017; Kim, Eur Radiol, 2018; Miwa, Abdom Radiol (NY), 2019. ' Azuma, Am J Surg, 2001; Xu, J Ultrasound Med, 2003; Cheon, World J Gastroenterol, 2009; Kim, Eur Radiol, 2016. ' Xu, J Ultrasound Med, 2003; Zielinskt, J Gastrointst Surg, 2008; Kim, Eur Radiol, 2018; Smereczynski, J Ultrason, 2020. 1 Zielinski, J Gastrointst Surg, 2008; Liu, J Gastroenterol Hepatol, 2018; Kim, Eur Radiol, 2018; Smereczynski, J Ultrason, 2020.

polyps in asymptomatic patients with false negative results on ultrasound could not be established, as these patients do not undergo surgery and are, therefore, impossible to identify. However, with a sensitivity of $56 \%$, numerous asymptomatic patients with potentially neoplastic polyps are expected to be overlooked. 
A total of $70 \%$ of the patients with a preoperative sonographic diagnosis of gallbladder polyps had nonneoplastic polyps at histopathology. Even though nonneoplastic polyps do not require removal by cholecystectomy, it would be remiss to state that all these patients were overtreated. Other factors, such as biliary symptoms or concomitant gallstones, can justify cholecystectomy and may have resulted in incidental nonneoplastic polyps on histopathology. These patients were excluded from the subgroup analysis to establish ultrasound performance in the subgroup of patients who had the most implications. In $25 \%$ of the patients who were specifically operated on for gallbladder polyps, polyps were falsely estimated as $\geq 1$ $\mathrm{cm}$, and $43 \%$ of the polyps $\geq 1 \mathrm{~cm}$ on ultrasound were nonneoplastic at histopathological analysis. The reason for this finding is a combination of the overestimation of polyp size by ultrasonography and the inaccuracy of the $1 \mathrm{~cm}$ threshold for neoplasia in general. In current clinical practice in which a sonographic polyp size $\geq 1 \mathrm{~cm}$ is the primary indicator for cholecystectomy in gallbladder polyps, the low specificity of routine preoperative ultrasonography contributes to the overtreatment of nonneoplastic gallbladder polyps. Even when considering secondary indications for cholecystectomy, such as symptoms, other malignant features, results of additional CT or MRI, and strong patient preference, considerable overtreatment remains.

The present study is not the only study to highlight the considerable overtreatment of gallbladder polyps, which underlines the need for improvements to ultrasound evaluation and patient selection for cholecystectomy for gallbladder polyps [20]. Multiple studies have outlined sonographic characteristics of gallbladder polyps that can be used to differentiate polyp type [6,9-12, 21]. The most recent European guidelines have sought to incorporate this development partly by excluding definite nonneoplastic polyps prior to polyp size assessment based on "comet tail" artifacts, which can be seen in adenomyomatosis or cholesterol polyps [4]. No other characteristics indicative of (non)neoplastic polyps or a structured overview of characteristics requiring evaluation and reporting are outlined in the guidelines. The present study illustrates that the characteristics that can predict polyp type, such as shape and echogenicity, are not routinely evaluated on ultrasound assessment (70\% of the reports did not list any polyp characteristics). Table 2 provides an overview of the imaging characteristics that recent literature suggests can be used to differentiate polyp types preoperatively. We hypothesize that standardizing ultrasound assessment to include these characteristics could help cre- ate uniformity in polyp evaluation and promote the use of detailed characteristics to differentiate polyp types. However, further research to assess the diagnostic accuracy of such ultrasound evaluation and its potential to improve patient selection for cholecystectomy is warranted.

Due to its low cost and wide availability, conventional ultrasound is likely to remain the primary modality for gallbladder (polyp) evaluation in the foreseeable future. Novel imaging techniques, such as contrast enhanced ultrasound, endoscopic ultrasound, CT, and MRI, may be used in difficult cases (e.g., in asymptomatic patients with inconclusive ultrasound imaging, technically challenging ultrasound imaging [obesity], or extended polyps) [4]. Although endoscopic ultrasound was not superior to conventional ultrasound in a Cochrane review, and contrast enhanced ultrasound, CT, and MRI have mainly shown positive results in large polyps $(>1 \mathrm{~cm})$, these modalities are worthy of further exploration for a more elaborate second-stage workup of gallbladder polyps [14, 2224].

The strengths of the present study include patient identification through PALGA and the inclusion of patients from academic and nonacademic hospitals, as routine clinical practice may differ. Although starting with histopathology could appear to be working backwards, in this manner, we were able to identify all patients with histopathologically proven gallbladder polyps, which is the gold standard for polyp identification and differentiation, from 4 hospitals over a 10 -year period, rather than only patients with polyps identified on ultrasound. Identifying patients with polyps that are not diagnosed preoperatively is paramount when determining the sensitivity of ultrasonography. The limitations of this study include the relatively small sample size and length of the study period, which resulted in potential variation in ultrasound equipment over the years. Unfortunately, the type of ultrasound equipment and assessor could not be considered in the diagnostic accuracy, as this information was lacking in the ultrasound reports. Last, not all ultrasound and pathology reports reported polyp size and number of polyps.

In conclusion, current routine ultrasound assessment has significant shortcomings in the diagnosis and evaluation of gallbladder polyps. With a low yield of neoplastic polyps on cholecystectomy and a low specificity of the sonographic $1 \mathrm{~cm}$ threshold for surgery, considerable overtreatment of nonneoplastic polyps in clinical practice should be considered. 


\section{Statement of Ethics}

This study did not involve human subjects and was granted exemption from requiring informed consent for use of anonymous patient data (METC Brabant, NW2017-37).

\section{Conflict of Interest Statement}

The authors have nothing to declare.

\section{Funding Sources}

This research did not receive any specific grant from funding agencies in the public, commercial, or not-for-profit sectors.

\section{Author Contributions}

The concept and design of work was performed by S.W., I.D., J.D., P.d.R., and C.v.L. Acquisition and analysis of the data was done by S.W., N.H., I.D., M.B., J.V., E.J.S.P., J.M., K.B., and H.v.d.L. Interpretation of the results was done by S.W., E.S.L., J.H., P.d.R.; C.v.L., S.W., and E.S.L. drafted the manuscript; N.H., I.D., M.B., J.V., E.J.S.P., J.M., K.B., J.H., P.d.R., J.D., and C.v.L. critically revised the manuscript. All authors approved the final version of the manuscript and agree to be accountable for all aspects of the work.

\section{References}

1 Aldouri AQ, Malik HZ, Waytt J, Khan S, Ranganathan $\mathrm{K}$, Kummaraganti S, et al. The risk of gallbladder cancer from polyps in a large multiethnic series. Eur J Surg Oncol. 2009 Jan; 35(1):48-51.

2 SAGES. Guidelines for the clinical application of laparoscopic biliary tract surgery. The Society of American Gastrointestinal and Endoscopic Surgeons; 2010.

3 European Association for the Study of the L. EASL clinical practice guidelines on the prevention, diagnosis and treatment of gallstones. J Hepatol. 2016 Jul;65(1):146-81.

4 Wiles R, Thoeni RF, Barbu ST, Vashist YK, Rafaelsen SR, Dewhurst C, et al. Management and follow-up of gallbladder polyps: joint guidelines between the European Society of gastrointestinal and abdominal radiology (ESGAR), European association for endoscopic surgery and other interventional techniques (EAES), International society of digestive surgery - European Federation (EFISDS) and European society of gastrointestinal endoscopy (ESGE). Eur Radiol. 2017 Sep;27(9): 3856-66.

5 Sun XJ, Shi JS, Han Y, Wang JS, Ren H. Diagnosis and treatment of polypoid lesions of the gallbladder: report of 194 cases. HBPD INT. 2004;3(4):591-4.

6 Zielinski MD, Atwell TD, Davis PW, Kendrick ML, Que FG. Comparison of surgically resected polypoid lesions of the gallbladder to their pre-operative ultrasound characteristics. J Gastrointest Surg. 2009;13(1):19-25.

7 Wennmacker SZ, van Dijk AH, Raessens JHJ, Van Laarhoven CJHM, Drenth JPH, De Reuver PR, et al. Polyp size of $1 \mathrm{~cm}$ is insufficient to discriminate neoplastic and nonneoplastic gallbladder polyps. Surg Endosc. 2019 May; 33(5):1564-71.

8 Xu A, Zhang Y, Hu H, Zhao G, Cai J, Huang A. Gallbladder polypoid-lesions: what are they and how should they be treated? A sin- gle-center experience based on 1446 cholecystectomy patients. J Gastrointest Surg. 2017 Nov;21(11):1804-12.

9 Xu HX, Yin XY, Lu MD, Liu L, Yue DC, Liu GJ. Comparison of three- and two-dimensional sonography in diagnosis of gallbladder diseases: preliminary experience. J Ultrasound Med. 2003 Feb;22(2):181-91.

10 Cheon YK, Cho WY, Lee TH, Cho YD, Moon JH, Lee JS, et al. Endoscopic ultrasonography does not differentiate neoplastic from non-neoplastic small gallbladder polyps. World J Gastroenterol. 2009 May 21; 15(19):2361-6.

11 Kim JS, Lee JK, Kim Y, Lee SM. US characteristics for the prediction of neoplasm in gallbladder polyps $10 \mathrm{~mm}$ or larger. Eur Radiol. 2016 Apr;26(4):1134-40

12 Liu XS, Chen T, Gu LH, Guo YF, Li CY, Li FH, et al. Ultrasound-based scoring system for differential diagnosis of polypoid lesions of the gallbladder. J Gastroenterol Hepatol. 2018 Jun;33(6):1295-9.

13 Casparie M, Tiebosch AT, Burger G, Blauwgeers $\mathrm{H}$, van de Pol A, van Krieken JH, et al. Pathology databanking and biobanking in The Netherlands, a central role for PALGA, the nationwide histopathology and cytopathology data network and archive. Cell Oncol. 2007;29(1):19-24.

14 Wennmacker SZ, Lamberts MP, Di Martino M, Drenth JP, Gurusamy KS, van Laarhoven CJ. Transabdominal ultrasound and endoscopic ultrasound for diagnosis of gallbladder polyps. Cochrane Database Syst Rev. 2018 Aug 15;8:CD012233.

15 Elmasry M, Lindop D, Dunne DF, Malik H, Poston GJ, Fenwick SW. The risk of malignancy in ultrasound detected gallbladder polyps: a systematic review. Int J Surg. 2016 Sep; 33 Pt A:28-35.

16 Babu BI, Dennison AR, Garcea G. Management and diagnosis of gallbladder polyps: a systematic review. Langenbecks Arch Surg. 2015 May;400(4):455-62.

17 French DG, Allen PD, Ellsmere JC. The diagnostic accuracy of transabdominal ultrasonography needs to be considered when managing gallbladder polyps. Surg Endosc. 2013 Nov;27(11):4021-5.

18 Lee JS, Kim JH, Kim YJ, Ryu JK, Kim YT, Lee JY, et al. Diagnostic accuracy of transabdominal high-resolution US for staging gallbladder cancer and differential diagnosis of neoplastic polyps compared with EUS. Eur Radiol. 2017 Jul;27(7):3097-103.

19 Lodhi A, Waite K, Alam I. The accuracy of ultrasonography for diagnosis of gallbladder polyps. Radiography. 2020 May;26(2):e52-5.

20 Pickering $\mathrm{O}$, Pucher $\mathrm{PH}$, Toale $\mathrm{C}$, Hand $\mathrm{F}$ Anand E, Cassidy S, et al. Prevalence and sonographic detection of gallbladder polyps in a Western European population. J Surg Res. 2020 Feb 24;250:226-31.

21 Kim SY, Cho JH, Kim EJ, Chung DH, Kim KK, Park YH, et al. The efficacy of real-time colour Doppler flow imaging on endoscopic ultrasonography for differential diagnosis between neoplastic and non-neoplastic gallbladder polyps. Eur Radiol. 2018 May;28(5):1994-2002.

22 Furukawa H, Kosuge T, Shimada K, Yamamoto J, Kanai Y, Mukai K, et al. Small polypoid lesions of the gallbladder: differential diagnosis and surgical indications by helical computed tomography. Arch Surg. 1998 Jul; 133(7):735-9.

23 Irie H, Kamochi N, Nojiri J, Egashira Y, Sasaguri $\mathrm{K}$, Kudo $\mathrm{S}$. High b-value diffusionweighted MRI in differentiation between benign and malignant polypoid gallbladder lesions. Acta Radiol. 2011 Apr 1;52(3):236-40.

24 Serra C, Felicani C, Mazzotta E, Gabusi V, Grasso V, De Cinque A, et al. CEUS in the differential diagnosis between biliary sludge, benign lesions and malignant lesions. J Ultrasound. 2018 Jun;21(2):119-26. 